\title{
ZENAIDA GUTIÉRREZ-VEGA, HISPANISTA CUBANA
}

POR

\author{
CARMEN ORTIZ \\ Instituto de Lengua, Literatura y Antropología \\ Centro de Ciencias Humanas y Sociales (CSIC)
}

El mundo del hispanismo internacional ha sido tradicionalmente considerado en España a partir de una especie de complejo de superioridad, que incluía una idea de tutelaje sobre las comunidades académicas latinoamericanas y que escondía a su vez una situación de inferioridad frente a éstas y, sobre todo, a las poderosas comunidades y departamentos de lenguas modernas y estudios hispánicos de las más potentes universidades norteamericanas y anglosajonas en general. Algo así como la salvaguarda de las esencias originales se convertía en un recurso de necesidad para las poco avanzadas líneas de investigación y visiones acerca de nuestra lengua y literatura que salían de las anquilosadas y mal dotadas universidades españolas.

El exilio posterior a la Guerra Civil nutrió a muchos de estos departamentos y universidades foráneas con profesores e investigadores españoles de primera línea, muchos de ellos todavía hoy no suficientemente reconocidos. Una de estas hispanistas españolas, exiliada de alguna manera, y profesora en el Hunter College de la City University of New York (CUNY), fue Soledad Carrasco Urgoiti, que murió en Nueva York el 5 de octubre de 20071. En ese mismo centro trabajaron también otras profesoras destacadas como Carmen $\mathrm{Zu}$ lueta y Zenaida Gutiérrez-Vega, amigas de Soledad Carrasco. La última vez que estuve con ésta en Madrid, y cuando nada hacía presagiar su muy cercana desaparición, hablamos de Zenaida Gutiérrez-Vega porque en su reciente (sería en realidad la penúltima de toda su vida) estancia en CUNY, Soledad había participado en el homenaje rendido por sus colegas y amigos a la profesora cubana, fallecida el 19 de febrero de 2007. Soledad se extrañó de que yo hubiese

\footnotetext{
1 García-Arenal, LXIII / 2 (Madrid, 2008): 261-265.
} 
leído algunas de las obras de su amiga y compañera, y me contó cómo ésta no había llegado a ver los ejemplares impresos de su último libro: José María Chacón y Calvo. Corresponsales cubanos, publicado en Madrid, que llegaron a Nueva York una semana después de su muerte ${ }^{2}$. De aquella entrevista salió el compromiso por mi parte de escribir una reseña de este libro póstumo. Para que me documentara al respecto, Soledad Carrasco me dio copia de su propia intervención en el homenaje rendido por su departamento universitario: «La España del siglo XX en la obra de Zenaida Gutiérrez Vega» ${ }^{3}$ y me regaló el librito del albacea testamentario de la profesora cubana, que fuera su compañero de muchos años en el Círculo de Cultura Panamericano, Carlos Ripoll: Zenaida Gutiérrez Vega In Memoriam ${ }^{4}$.

Zenaida Gutiérrez-Vega (Unión de Reyes, Cuba, 1924; Nueva York, EE.UU., 2007) terminó su licenciatura en Letras en la Universidad de La Habana en 1950, donde obtuvo también su título de doctora con una tesis sobre La vorágine de José Eustasio Rivera. Tras una estancia en Europa en 1956, consiguió la cátedra de historia del arte en la Universidad Central de las Villas y dio a conocer sus primeras investigaciones sobre Martí. Tras el triunfo de la revolución, abandona Cuba en 1962, aprovechando una beca del Instituto $\mathrm{Cu}$ bano de Cultura Hispánica para una estancia en Madrid, en cuya Universidad Central defiende una nueva tesis doctoral, dedicada a la Vida y obra de José María Chacón y Calvo que daría lugar a su primer libro, José María Chacón y Calvo, hispanista cubano publicado en $1969^{5}$, precisamente el año de su muerte en La Habana. Durante los años de preparación de su tesis, la joven investigadora se hospedó en el piso de la calle General Pardiñas de Madrid, donde había vivido Chacón y que éste mantenía a pesar de que nunca volvió a España, después de su última partida en 1957, lo que es prueba de la confianza que le demostró Chacón. Esta absoluta confianza se demuestra en el acceso que Gutiérrez-Vega tuvo al archivo del diplomático y polígrafo cubano, que se plasmó en la publicación de buena parte de la correspondencia que mantuvo con personalidades punteras de la vida cultural española y latinoamericana del floreciente primer tercio del siglo XX. Como nos explica la propia autora, esta estrecha sintonía fructificó en importantes aportaciones:

«Deseosa de dar a conocer mejor la valiosa obra del hispanista cubano, ideé el modo de divulgarla a través de sus corresponsales. Le comuniqué mi deseo y, en carta de 4 de noviembre de 1966, me escribió: "Tiene usted carta blanca en esta idea suya

2 Gutiérrez-Vega, 2006.
3 Carrasco Urgoiti, s. p.
4 Ripoll, 2007a.
5 Gutiérrez-Vega, 1969. 
de los epistolarios. Todo lo hará usted bien y yo no tengo sino expresarle una vez más mi gratitud". Así surgieron mis libros publicados sobre José María en la Fundación Universitaria Española de Madrid: Epistolario Alfonso Reyes-José M. Chacón (1976), Fernando Ortiz en sus cartas a José M. Chacón (1982), Estudio bibliográfico de José M. Chacón (1982), Corresponsales españoles de José M. Chacón (1986) y mis extensas monografías Pedro Henríquez Ureña, maestro continental (Cartas a José M. Chacón y Calvo), en RevIb, n. ${ }^{\circ}$ 94, 1976 y Max Henríquez Ureña. Cartas de un maestro (a José M. Chacón y Calvo), $\mathrm{CH}$. Madrid, n. ${ }^{\circ} 380,1982 »^{6}$.

Cuando Gutiérrez-Vega decide abandonar España rumbo a los Estados Unidos en 1967, se lleva consigo los archivos de Chacón y Calvo para continuar con sus investigaciones. Su primer destino norteamericano es la Universidad de San Luis, Missouri, de la que pasó a la State University of New York, para recalar definitivamente, en 1971, en el Hunter College de la City University of New York en la que se jubilará en 1998 y a la que seguirá ligada como Profesora Emérita hasta el final de sus días.

En Nueva York entrará en relación con Victoria Kent, a través de la poeta española Carmen Conde. Precisamente unas conversaciones con ésta, mantenidas conjuntamente con Marie-Lise Gazarian-Gautier, dieron lugar al libro, Carmen Conde: de viva voz (1992), que recoge además de las conversaciones sendos ensayos de las autoras, el de Gutiérrez-Vega dedicado al análisis del lenguaje poético de Carmen Conde en relación con su vivencia de la Guerra Civil ${ }^{7}$. Las circunstancias del exilio de la política republicana, asentada definitivamente en Estados Unidos, gracias al patrocinio de Louise Crane, la acercan a la investigadora cubana como «la persona ideal para confiarle el tesoro de sus archivos $\rangle^{8}$, convirtiéndola así de nuevo en la depositaria de la memoria personal y los archivos de una intelectual. Zenaida Gutiérrez-Vega culminará, como había hecho con Chacón y Calvo, esta relación personal con la publicación de una importante biografía: Victoria Kent: una vida al servicio del humanismo liberal ${ }^{9}$. Este libro - además de reivindicar una figura que había sido vapuleada por los republicanos izquierdistas, por el franquismo y no bien comprendida por los movimientos feministas - gracias al manejo de la correspondencia y otros documentos privados, proporciona valiosos datos acerca de sus relaciones con el exilio republicano español y también con la clandestina oposición en el interior, a los que Victoria Kent y Louise Crane prestaron voz y visibilidad internacional a través de su revista Ibérica ${ }^{10}$.

6 Gutiérrez-Vega, 2006: 4-5.

7 Gutiérrez-Vega y Gazarian-Gautier, 1992: 162-169.

8 Ripoll, 2007a: 14.

9 Gutiérrez-Vega, 2001.

10 Carrasco Urgoiti, s. p. 
Finalmente, Gutiérrez-Vega recibirá también correspondencia original, libros y otros documentos de una tercera figura académica muy diferente, el español Pedro Sainz Rodríguez, que fue durante un largo periodo director de la Fundación Universitaria Española y, como tal, patrocinador de los libros editados por Gutiérrez-Vega con la correspondencia de Chacón y Calvo ${ }^{11}$. Estos importantes depósitos documentales fueron legados por disposición testamentaria de la profesora cubana a su colega Carlos Ripoll, Profesor Emérito de Queens College en CUNY.

En definitiva, la diáspora y la controversia política que marcó la vida de los personajes involucrados en esta trama de amistad y estudio, basada en el amor hacia Cuba y España y en el interés por conocer su historia y su literatura, se ha mantenido más allá de la muerte de sus protagonistas y así los documentos, la biblioteca, las fotografías (a las que era un gran aficionado), las cartas y los diarios acumulados a lo largo de su vida por José María Chacón y Calvo se conservan dispersos y repartidos entre los ámbitos que fueron significativos en su trayectoria intelectual, política y humana. Una parte de sus libros y papeles dejados en la que fuera su casa madrileña se encuentran en la Biblioteca del antiguo Instituto de Cooperación Iberoamericana (hoy AECI) en Madrid. En La Habana se conserva otra parte importante, el archivo de la última etapa de su vida, depositado en la antigua Biblioteca de La Sociedad Económica de Amigos del País, sede actual del Instituto de Literatura y Lingüística de Cuba. Finalmente, los intelectuales del exilio cubano en los Estados Unidos han sido los depositarios de los documentos reunidos por Chacón durante sus largos periodos de estancia en España, que Zenaida Gutiérrez-Vega se llevó consigo al abandonar España. Como veremos, estos tres sectores están igualmente involucrados en el rescate histórico de la figura y la obra del escritor cubano.

José María Chacón y Calvo (Santa María del Rosario, La Habana, 29-10-1892; La Habana, 11-11-1969), miembro de una familia distinguida, estudió Derecho y Letras en la Universidad de La Habana y en 1918 consiguió su primer empleo como Secretario de la Legación de Cuba en Madrid, donde permaneció hasta 1936; volvería luego intermitentemente, hasta 1957 en que se produjo su última estancia en España. Este destino diplomático le dará cobertura para desarrollar su verdadera vocación: la literatura, y la oportunidad de entrar en contacto con los círculos más vanguardistas de la cultura española del momento, que se movían en torno a la Residencia de Estudiantes y el resto de las iniciativas patrocinadas por los intelectuales ligados a la Institución Libre de

11 La lista bibliográfica con los trabajos publicados por Zenaida Gutiérrez-Vega puede consultarse en Ripoll, 2007a. 
Enseñanza: La Junta para Ampliación de Estudios, el Centro de Estudios Históricos, etc. De hecho, ya antes de su venida a España, Chacón había entablado relación con Ramón Menéndez Pidal, a raíz de uno de los primeros estudios publicados por el erudito cubano, y su primera incursión en el folclore: Romances tradicionales en Cuba ${ }^{12}$, y posteriormente, como Director de Cultura del Ministerio de Educación, desempeñará un papel importante en la organización del viaje y la estancia del filólogo español en Cuba a principios de 1937, viaje que será posteriormente rememorado por Chacón ${ }^{13}$ y que demuestra su implicación, junto con Fernando Ortiz, en el apoyo a los intelectuales republicanos empujados al exilio ${ }^{14}$.

La figura de José María Chacón y Calvo sólo recientemente ha comenzado a ser valorada en Cuba, y su legado empieza ahora a ser objeto de atención por parte de las instituciones e intelectuales de la isla. Pero mucho antes de que esto ocurriera, Zenaida Gutiérrez-Vega dio a conocer públicamente no sólo la obra erudita, literaria y crítica del diplomático, sino que puso en primer plano su importancia como figura relevante en el entramado cultural e intelectual de la Cuba republicana, y la profundidad y largueza de las relaciones que estableció personalmente $-\mathrm{y}$ que ayudó generosamente a que se convirtieran en institucionales - con los movimientos más vanguardistas de la efervescente cultura española del primer tercio del siglo XX. Gracias a Zenaida Gutiérrez-Vega, y a su trabajo de edición de una parte muy significativa de la correspondencia de Chacón y Calvo, podemos tener acceso a detalles relevantes de cómo se desenvolvía la actividad literaria y política del Madrid de los años anteriores a la Guerra Civil, en el que, junto a la generación de poetas del 27, a artistas y músicos reputados, circulaban también científicos y humanistas de primera línea, y dónde encontraban acomodo casi familiar figuras fundamentales de la intelectualidad latinoamericana. El libro dedicado por Gutierréz-Vega al epistolario mantenido entre el mexicano Alfonso Reyes y José M. ${ }^{a}$ Chacón es una espléndida muestra de esta última realidad ${ }^{15}$, pero también la amistad con Pedro y Max Henríquez Ureña, darían lugar a sendos ensayos ${ }^{16}$.

Cómo es lógico, en la intensa actividad epistolar mantenida por Chacón durante su estancia en España tuvo un lugar especial la correspondencia con sus colegas y amigos cubanos. En este sentido, es muy interesante la que cruza

12 Chacón y Calvo, 1914.

13 Chacón y Calvo, 1961.

14 Puig-Samper y Naranjo, 9 (Praga, 2001): 199-213.

15 Gutiérrez-Vega, 1976a.

16 Gutiérrez-Vega, XLII / 94 (Pittsburgh, 1976b): 103-114; 380 (Madrid, febrero 1982b): 298-343. 
con Fernando Ortiz ${ }^{17}$, que demuestra la enorme actividad desplegada por Chacón en Madrid para apoyar las iniciativas patrocinadas por el antropólogo cubano, como la Institución Hispano-Cubana, con el objetivo de establecer y mantener vínculos institucionales que facilitaran el contacto y la presencia fluida de la ciencia y la cultura española en Cuba.

Pero, lógicamente, las cartas que despertaron mayor interés en España $-\mathrm{y}$ también fuera- son las que Chacón conservaba en su archivo personal, firmadas por los que fueran sus amigos, historiadores, poetas y escritores, durante los años en que vivió en Madrid, cuya nómina incluye los nombres de Salvador Dalí, Manuel de Falla, Rafael Alberti y Federico García Lorca, pero también a Pío Baroja, Américo Castro, Ramón Gómez de la Serna y Ramón Menéndez Pidal, entre otros.

De entre ellas, las de Federico García Lorca, con poemas dedicados a Chacón e ilustradas con dibujos del poeta, firmadas a veces por «Federico rex»o «Federico rex de Andalucía», son un producto muy emotivo de la amistad y la confianza íntima entre los dos. Este importante epistolario, dado a conocer inicialmente por Gutiérrez-Vega, dio lugar posteriormente a nuevos análisis, como el que hacen González Montes y Tinnell de las cartas de Chacón ${ }^{18}$. Por otro lado, las cartas de García Lorca a Chacón en Madrid, junto con otros documentos, fotografías, etc., de su archivo, conservados en el legado de Gutiérrez-Vega, han vuelto a ser transcritos y editados en un libro reciente firmado por Carlos Ripoll ${ }^{19}$. José M. Chacón fue, asimismo, el artífice principal de la estancia en Cuba de García Lorca entre febrero y junio de 1930, invitado por la Institución Hispano-Cubana para dar conferencias en La Habana y otras ciudades de la isla. Una de las cosas que más huella dejaría al poeta granadino en este viaje importante para su biografía fue la amistad trabada con los hermanos Loynaz. Enrique Loynaz Muñoz fue, a su vez, uno de los amigos más íntimos de José María Chacón, quien guardó en su archivo una serie de poemas suyos inéditos, que fueron dados a la luz por Zenaida Gutiérrez-Vega ${ }^{20}$.

La tupida red de correspondencias, ensayos críticos, prólogos y ediciones llevada a término por Chacón y Calvo, y los documentos y papeles acumulados por él a lo largo de su fructífera vida, no sólo se convirtieron en el objeto preferente del trabajo de Gutiérrez-Vega. Siguiendo de alguna manera su estela, otros historiadores y literatos, tanto en Cuba como fuera de la isla, han pu-

17 Gutiérrez-Vega, 1982a.

18 González Montes y Tinnell, 1 / 1 (Washington, 2000): 65-85.

19 Ripoll, 2007b.

20 Gutiérrez-Vega, IX / 10 (Madrid, 1981): 107-126. 
blicado recientemente otras partes de la correspondencia del erudito cubano y están empeñados en revalorizar su figura y su trayectoria humana e intelectual. Así, por ejemplo, en la propia Habana se observa a partir de la década de 1990 un movimiento a favor de la recuperación y conservación de su obra $^{21}$. Entre otras iniciativas, se comenzó un proyecto para catalogar los documentos y fondos bibliográficos conservados por la Biblioteca de la AECI en Madrid y el Instituto de Literatura y Lingüística de La Habana; se le dedicó incluso la edición de un sello de correos en $1992^{22}$ y existe el propósito de crear una Fundación José María Chacón dedicada al que fuera, durante tantos años, Director de la Academia Cubana de la Lengua y del Ateneo de La Habana, fundador de la Revista Cubana, colaborador asiduo de El Fígaro, El Mundo, Diario de la Marina, Revista Bimestre Cubana, etc., y que sobrevivió sus últimos años en medio de la penuria, ocupando como último puesto la cátedra de literatura cubana en la Universidad Católica de Villanueva, entre 1946 y 1961.

Aparte del folleto publicado en $\mathrm{Miami}^{23}$, en Cuba se está llevando a cabo un proyecto editorial de envergadura para rescatar los diarios de Chacón, género del que en vida del autor ya hubo una muestra destacable referida a los días dolorosos de la muerte de su madre (a la que siempre estuvo muy unido) ${ }^{24}$. Hasta ahora se han editado, de forma conjunta, las dos primeras partes: Primer diario de España y Diario del joven desconocido, en un libro con presentación y notas de Virgilio López Lemus, que incluye el manuscrito de cada uno de estos dos diarios, correspondientes a la época pasada por Chacón en la legación cubana en España ${ }^{25}$. El primero de ellos abarca del 3 de octubre de 1918 al 8 de mayo de 1922, mientras que el segundo comprende las anotaciones hechas entre el 3 de febrero y el 20 de julio de 1923. Estos diarios tienen similitudes estilísticas con el escrito en ocasión de la muerte de su madre, presentan una prosa poética que muestra, tanto las propias angustias existenciales del autor, como sirve para evocar paisajes y experiencias personales en sus viajes por los pueblos y ciudades de España en el primero, y para testimoniar la presencia de algunos amigos y personajes importantes en su vida, como José Luis Galbe, su «hermanito menor» y guía de sus rutas por el Pirineo, o el poeta Enrique Loynaz ${ }^{26}$.

21 Bueno, 1994; Chacón y Calvo, 1998.

22 Ripoll, 2007a.

23 Aparicio Laurencio, 1987.

24 Chacón y Calvo, 1953.

25 Chacón y Calvo, 2007.

26 Iturria Savón, 2008. 
Sin embargo, teniendo en cuenta los momentos y acontecimientos dramáticos que son su objeto y la inmediatez que supone su traslado a un diario, puede resultar más interesante (sin duda lo será para el lector español) desde el punto de vista histórico la segunda entrega (cronológicamente hablando): el Diario intimo de la Revolución Española, editado en 2006 por el Instituto de Literatura y Lingüística de Cuba, bajo la supervisión del investigador José Antonio Michelena y con presentación y notas de Nuria Gregori Torada ${ }^{27}$. El diario recoge lo que Chacón y Calvo vivió en Madrid entre el 18 de julio de 1936 (las anotaciones comienzan a los cuatros días de producirse la sublevación nacionalista) y el 2 de noviembre del mismo año, en que vuelve a Cuba, acuciado por la enfermedad de su madre y espantado por el gravísimo estado de violencia en que se veía inmerso, e incluye al final el epistolario de Chacón con destacados intelectuales residentes en Madrid por aquellos días. La polarización política que se vivió en Cuba a raíz de la guerra española y la asimilación de Chacón y Calvo con el bando falangista, debido a su público catolicismo y a su colaboración con el Diario de la Marina —incondicionalmente posicionado, de la mano de su director José Ignacio Rivero y Alonso, a favor de Fran$\mathrm{Co}^{28}$ - son elementos que han pesado siempre sobre los juicios y la consideración de su figura, y han llevado a su encasillamiento como un personaje conservador e incluso reaccionario. Sin embargo, la publicación de este diario arroja muchas más luces y matices sobre la figura de Chacón y su compromiso social, porque en él aparecen los denodados esfuerzos por ayudar y proteger a sus amigos y conocidos, y se aprecia asimismo el mantenimiento de su lealtad hacia personas cuyo republicanismo e izquierdismo no puede ponerse en duda (por ejemplo, Pablo de la Torriente).

No obstante, quedan inéditos todavía, además de la mayor parte de su enorme epistolario posterior a su definitiva vuelta a Cuba, el diario que siguió escribiendo entre 1959 y 1969, en el momento crepuscular de su vida, cada vez más distante y aislado, cuando fue viendo desaparecer, hacia el exilio en parte, a la mayoría de sus amistades y colaboradores antiguos. Pero, como ha señalado Miguel Iturria en su reseña de los diarios publicados de Chacón, el dar al público este interesante material, «exigirá un voto contra la censura pues aún viven los protagonistas de esta etapa convulsa que Chacón y Calvo valoró desde su casona de El Vedado, donde murió en la miseria y el abandono» ${ }^{29}$.

Si más arriba se ha dicho que toda esta actividad recuperadora de la figura y la obra de Chacón y Calvo hay que considerarla como una secuela de la ini-

27 Chacón y Calvo, 2006.

28 Figueredo Cabrera, 10 (Madrid, 2007): 1-18.

29 Iturria, 2008. 
ciada por Zenaida Gutiérrez-Vega, el último libro de ésta coincide también - paradójicamente desde el exilio cubano y volviendo al patrocinio editorial de una institución española - en el abordaje del legado cubano contenido en el archivo que Chacón formó en Madrid y que es conservado por los herederos intelectuales de Gutiérrez-Vega en Nueva York. El último libro de Zenaida Gutiérrez-Vega: José María Chacón y Calvo. Corresponsales cubanos (2006) recoge un corpus epistolar obtenido de los archivos de Chacón y del propio de la autora, y abarca una amplia cronología entre 1913 y 1969; es decir desde la etapa de estudiante en la Universidad, hasta el año de la muerte del autor. Sin embargo, la mayor parte de las cartas están fechadas entre 1918 y 1936, que son los años en que Chacón vive en España y, por tanto en los que el escribir se mantiene como la forma normal de comunicación con sus compatriotas. El contacto por escrito se mantuvo después con muchos de ellos y varios de los corresponsales enviaron sus cartas a Gutiérrez-Vega para su proyecto, pero otras muchas quedaron en la Habana entre los papeles de Chacón ${ }^{30}$.

Igualmente puede decirse que los 23 corresponsales que se incluyen en el corpus suponen una amplísima representación de la historia y la cultura cubanas desde el final del siglo XIX hasta más allá de la mitad del XX y que entre ellos aparecen figuras señeras de la política (Eliseo Giberga, Cosme de la Torriente, Manuel Sanguily, Orestes Ferrara, etc.), pero también (y sobre todo) de la literatura (Agustín Acosta, Luisa Pérez de Zambrana, Dulce María Loynaz, Emilio Ballagas, Regino Boti, Jorge Mañach, José Antonio Ramos, Alfonso Hernández Catá, Luis Felipe Rodríguez, etc.). Las generaciones más influyentes de la historia intelectual cubana están, por tanto, representadas en esta correspondencia, comenzando por el Autonomismo y el Separatismo. Con todo, el grueso del libro lo constituyen las cartas de dos personajes muy diferentes, el filósofo separatista y colaborador de Martí, Enrique José Varona, de quien Chacón fue alumno en la Universidad de La Habana, por un lado, y, por el otro, la correspondencia íntima del amigo poeta Agustín Acosta Bello (1886-1979). Como en los otros libros de cartas de Chacón editados por Gutiérrez-Vega, los distintos corresponsales aparecen por orden alfabético (y sus cartas ordenadas por fechas) y sus misivas van encabezadas por una semblanza breve de los mismos, debida al mismo Chacón, tomada de alguno de sus múltiples artículos de prensa, reseñas o críticas publicadas sobre los distintos autores.

En suma, este último libro de Zenaida Gutiérrez-Vega, José María Chacón y Calvo. Corresponsales cubanos, puede ser considerado, por un lado, como el

30 Gutiérrez-Vega, 2006: 8. 
cierre del compromiso vital e intelectual mantenido durante toda la vida con su maestro cubano, pero, por otro, muestra también una confluencia entre la labor de la profesora de Nueva York y la de otros investigadores de la isla y de fuera, $y$, en este sentido, significa, sin duda, un gran aporte para el mundo académico y universitario que, en Cuba, está construyendo críticamente su pasado más inmediato.

\section{BIBLIOGRAFÍA}

Aparicio Laurencio, A. (ed.), Don José M. Chacón y Calvo en su correspondencia, Miami, Universal, 1987.

Bueno, Salvador (comp.), Cubanidad y españolidad de José María Chacón y Calvo, La Habana, Editorial Letras Cubanas, 1994.

Carrasco Urgoiti, M. Soledad, La España del siglo XX en la obra de Zenaida Gutiérrez-Vega, sin publicar.

Chacón y Calvo, José María, Romances tradicionales en Cuba (Contribución al estudio del folklore cubano), La Habana, Imprenta el Siglo XX, 1914.

Chacón y Calvo, José María, Diario en la muerte de mi madre, La Habana, Imp. P. Fernández, 1953.

Chacón y Calvo, José María, Los días cubanos de Menéndez Pidal, La Habana, 1961.

Chacón y Calvo, José María, Visión de autores españoles, La Habana, Centro Cultural de España-Editorial José Martí-Instituto Cubano del Libro, 1998.

Chacón y Calvo, José María, Diario íntimo de la revolución Española, La Habana, Instituto de Literatura y Lingüística, 2006.

Chacón y Calvo, José María, Primer diario de España y Diario del joven desconocido, La Habana, Instituto de Literatura y Lingüística, 2007.

Figueredo Cabrera, Katia, «Cuba en la estrategia cultural de la España franquista (1945-1958)», Pensamiento y Cultura, 10 (Chía, Cundinamarca, 2007): 1-18.

García-Arenal, Mercedes, «María Soledad Carrasco Urgoiti (1922-2007)», Revista de Dialectología y Tradiciones Populares, LXIII / 2 (Madrid, 2008): 261-265.

González Montes, Yara y Tinnell, Roger, «Epistolario de José María Chacón y Calvo a Federico García Lorca», Iberian and Latin American Studies, 1/1 (Washington, 2000): 65-85.

Gutiérrez-Vega, Zenaida, José María Chacón y Calvo, hispanista cubano, Madrid, Ediciones de Cultura Hispánica, 1969.

Gutiérrez-Vega, Zenaida, Epistolario Alfonso Reyes José-M. Chacón, Madrid, Fundación Universitaria Española, 1976a. 
Gutiérrez-Vega, Zenaida, «Pedro Henríquez Ureña, maestro continental», Revista Iberoamericana, XLII / 94 (Pittsburg, 1976b): 103-114.

Gutiérrez-Vega, Zenaida, «La poesía inédita de Enrique Loynaz Muñoz», Anales de Literatura Hispanoamericana, IX / 10 (Madrid, 1981): 107-126.

Gutiérrez-Vega, Zenaida, Fernando Ortiz en sus cartas a José M. ${ }^{a}$ Chacón (1914-1936, 1956), Madrid, Fundación Universitaria Española, 1982a.

Gutiérrez-Vega, Zenaida, «Max Henríquez Ureña: cartas de un maestro», Cuadernos Hispanoamericanos, 380 (Madrid, febrero 1982b): 298-343.

Gutiérrez-Vega, Zenaida, Victoria Kent: una vida al servicio del humanismo liberal, Málaga, Universidad de Málaga, 2001.

Gutiérrez-Vega, Zenaida, José María Chacón y Calvo. Corresponsales cubanos, Madrid, Fundación Universitaria Española, 2006.

Gutiérrez-Vega, Zenaida y Gazarian-Gautier, Marie-Lise, Carmen Conde: de viva voz, Nueva York, Senda Nueva Ediciones, 1992.

Iturria Savón, Miguel, «Los diarios de Chacón y Calvo (final)», CubaNet. http://www.cubanet.org/CNews/y08/julio08/02cronica1.html, 2008. Consultada el 28-4-2009.

Puig-Samper, Miguel Ángel y Naranjo, Consuelo, «La acogida del exilio español en Cuba: Fernando Ortiz y la Institución Hispanocubana de Cultura», Ibero-Americana Pragensia - Suplementum, 9 (Praga, 2001): 199-213.

Ripoll, Carlos, Zenaida Gutiérrez Vega In Memoriam, Nueva York, Editorial Dos Ríos, 2007a.

Ripoll, Carlos, Cuba en Lorca, Nueva York, Editorial Dos Ríos, 2007b.

Fecha de recepción: 18-1-2008

Fecha de aceptación: 5-3-2008 